\section{EVALUATION OF THE DANGER SIGNAL HMGB1 AS A POTENTIAL BIOMARKER IN JUVENILE IDIOPATHIC ARTHRITIS (JIA): A PRELIMINARY STUDY USING THE NOVEL BIOBANK JABBA}

Hanna Schierbeck, ${ }^{1}$ Rille Pullerits, ${ }^{2}$ Chris Pruunsild ${ }^{3}$ Marie Fischer, ${ }^{4}$ Dirk Holzinger, ${ }^{5}$ Karin Palmblad, ${ }^{1}$ Ann-Charlotte Aveberger, ${ }^{1}$ Erik Sundberg, ${ }^{1}$ Helena Erlandsson Harris ${ }^{1}$ Department of Women's and Children's Health, Rheumatology Unit, Karolinska Institute, Stockholm, Sweden; 'Department of Rheumatology and Inflammation Research, University of Gothenburg, Gothenburg, Sweden; 'University of Tartu, Tartu University Children's Hospital, Tartu, Estonia; ${ }^{4}$ Department of Medicine, Rheumatology Unit, Karolinska Institute, Stockholm, Sweden; ${ }^{5}$ Institute of Immunology, University of Münster, Münster, Germany

10.1136/annrheumdis-2011-201230.23

Background and objectives The endogenous danger signal High Mobility Group Box protein 1 (HMGB1) promotes inflammation. HMGB1 has been implicated as a mediator of RA while there are no reports describing its presence in JIA patient samples. HMGB1 is aberrantly expressed in the synovitis of RA patients, intraarticular injection of HMGB1 induces arthritis in mice and HMGB1 blockade has beneficial effects in several different experimental disease models, including arthritis.

In this study, the aim was to determine whether HMGB1 is extracellularly increased during JIA and, if so, to correlate the HMGB1 levels both with other more well-described laboratory parameters and with clinical parameters such as age at onset and disease duration. This type of descriptive study forms the basis for the evaluation of HMGB1 as a biomarker of inflammatory activity during JIA and as a potential therapeutic target. The newly established JIA biobank JABBA coupled to a clinical register gives us a unique opportunity to study JIA pathogenesis.

Materials and methods Plasma and synovial fluid (SF) was collected from 23 patients with JIA (median age $12(2-18)$ ) at Astrid Lindgren Children's hospital, Stockholm, Sweden and at Tartu University Children's Hospital, Estonia. Samples were analysed 
by ELISA and Cytometric bead array (CBA) to measure levels of HMGB1, MMP-3, sRAGE, IL-12p70, TNF, IL-10, IL-6, IL-1 $\beta$, IL-8, MCP-1, IP-10, RANTES, IFN $\gamma$, IFN $\alpha$ and IL-17A.

Results Increased HMGB1 levels were recorded in SF as compared to plasma from JIA patients. Highest levels of HMGB1 were recorded in patients with a disease onset in early age (before age 10), while no correlation between the HMGB1 levels and disease duration was evident. In contrast, both S100 and IL-8 levels correlated with disease duration being highest during early stages of disease. MMP-3, a marker of cartilage destruction, was higher in patients with late disease onset which indicates similarities with RA.

Conclusion The increased levels of HMGB1 in inflamed joints of JIA patients warrants further studies of HMGB1 as a biomarker for inflammatory activity and as a target for therapy. The correlation of HMGB1 levels with age at disease onset and continuously high levels irrespective of disease duration indicates, together with the decreasing levels of S100 and IL-8 during the disease course, that the inflammatory process in JIA evolves over time. Different mediators might thus be of varying importance during the disease progression. The authors conclude that the role of HMGB1 in the pathogenesis of JIA deserves further investigation. 